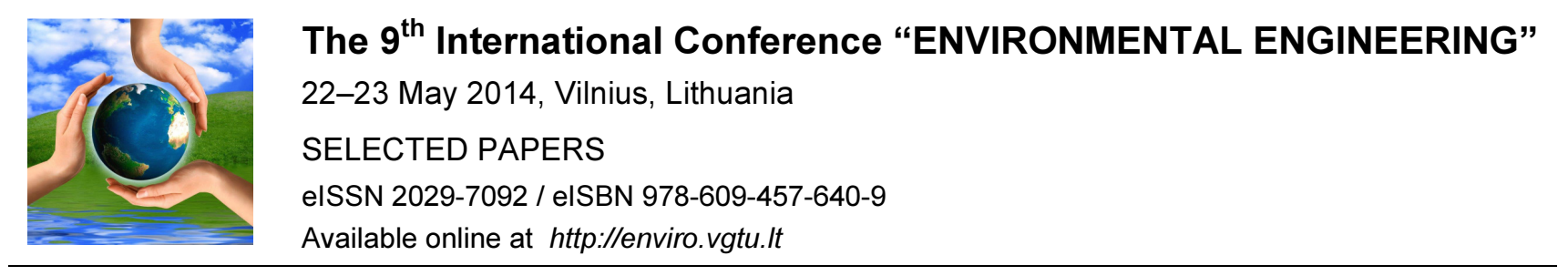

Section: Technologies of Geodesy and Cadastre

\title{
Handling of control measurements with three-dimensional visualization of geometric changes
}

\author{
Krzysztof Bojarowski \\ Institute of Geodesy, University of Warmia and Mazury, 10-720 Olsztyn, Poland
}

\begin{abstract}
Measurement and computation of geometric indices indicating the values of distortions, displacements and deviations is of major importance in control work. The application of three-dimensional object modelling in combination with the spatial analysis functions enables a broadening of the methods of interpreting the results of these determinations. This paper presents patterns of action for different control measurement, result handling and adopted visualization methods.
\end{abstract}

Keywords: digital terrain model; numerical map; analysis of alteration.

\section{Introduction}

Handling of displacement measurement results usually included, in the traditional form, numerical compilations and different types of graphs, profiles of vertical displacements and sketches and maps of horizontal displacements [1], [2]. New possibilities of representation and interpretation of displacement determination results have emerged in connection with the development of survey methods, where laser scanning or mirrorless tacheometric surveying can be examples. Simultaneous determination of displacement components in the three primary directions allowed their three-dimensional representation [3], including the form of a three-dimensional model. Such compilations were, however, relatively onerous until recently, because they lacked automation of performed tasks. These possibilities have developed in recent years due to specialized computer systems designed for the processing and visualization of spatial information. Among these systems, AutoCAD Civil 3D and ArcGIS with the 3D Analyst overlay offer particularly useful functions. These systems combine functions enabling geodetic handling of survey data, including a so-called "point cloud", with spatial analysis and three-dimensional modelling [4], [5], [6], [7].

\section{Assumptions of the proposed method}

Among the tasks carried out using CAD systems and spatial information systems, compilations in 3D space are of growing importance. In most cases, and particularly for engineering structures, the possibility of this type of analysis is conditioned by prior creation of a digital model [5]. The experience of recent years has shown that there is not only a demand for systems containing 3D information, but also an urgent need to speed up work in this area, particularly for the needs of designers and users of digital maps. Space modelling is also necessary in situations where the topography is of economic or natural importance or poses different kinds of risks (flood monitoring, landscaping, examination of the Earth's crust movements, etc.). DTM application broadens the possibilities of obtaining synthetic information about structures and phenomena also in many cases concerning the design and operation of engineering and hydraulic engineering structures [7].

The general diagram of processing survey results for engineering structures is shown in Figure 1. According to this diagram, the source information are geodetic survey results enabling the determination of the position of the points on the examined structure in a three-dimensional system at a specific time $\mathrm{X}(\mathrm{t}), \mathrm{Y}(\mathrm{t}), \mathrm{Z}(\mathrm{t})$. Sets of observation results can also be used to compute geometric indices describing the shape of the structure or its deformations. The computed indices for selected points can then be interpolated to the surface of the whole structure.

Corresponding author: Krzysztof Bojarowski. E-mail address: kboj@uwm.edu.pl

http://dx.doi.org/10.3846/enviro.2014.194

(C) 2014 The Author. Published by VGTU Press. This is an open-access article distributed under the terms of the Creative Commons Attribution License, which permits unrestricted use, distribution, and reproduction in any medium, provided the original author and source are credited. 


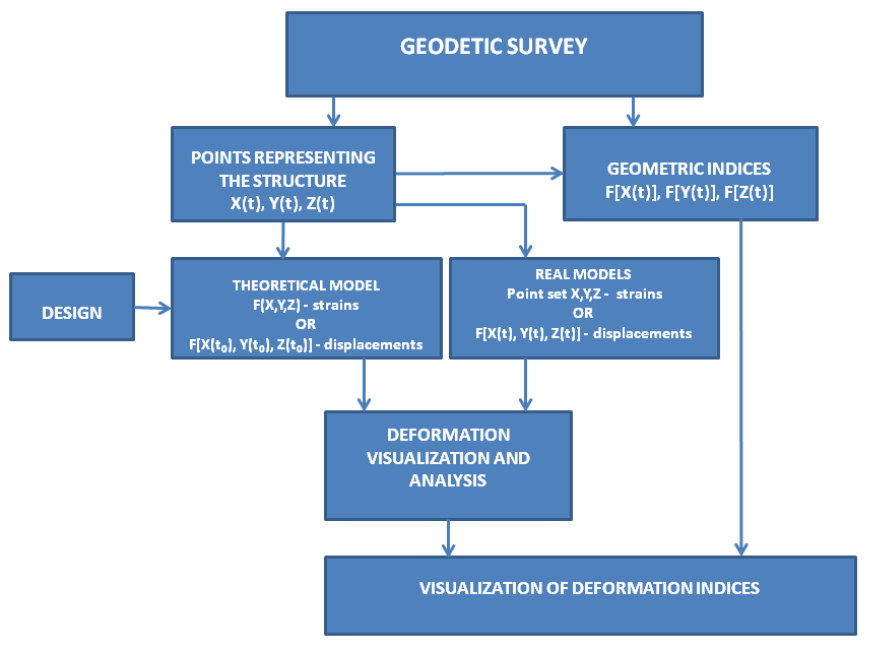

Fig. 1. General processing diagram for survey results

Coordinate sets computed based on survey results, which can be ordered as a function of time, are used to create a theoretical model or, in displacement determination, to generate a model in the period to. In many cases, especially when the analysed structures have an irregular shape, they are modelled by the creation of TIN or GRID surfaces. It should be pointed out that faithful reflection of the shape of a structure depends on the selection of the survey points [6]. The person conducting the survey decides during its performance on the recording of a point with a specific position, simultaneously taking care that the structure generalization does not cause greater errors than the assumed accuracy of the created model. In modern survey methods, points are recorded automatically (e.g. laser scanner) and their positions are determined by the instrument parameter settings. The next stage of actions shown in Figure 1 is an analysis of the created spatial models. The course and results of this stage largely depend on the functions offered by the computer system. These are most often all kinds of surface analysis results and generated profiles and sections.

\section{Applied coordinate systems}

Examination of geometric changes of structures involves the establishment of a geodetic control network which is the reference system and the choice of a method allowing the measurement of linear and angular values with the assumed accuracy [1], [2]. During the survey, each station forms a local coordinate system and, depending on the survey method, the result can be values defining a point's position in an orthogonal $(\mathrm{x}, \mathrm{y}, \mathrm{z})$, cylindrical $(\mathrm{r}, \varphi, \mathrm{z})$ or spherical system $(\mathrm{r}, \varphi, \lambda)$. Based on the survey results in two successive epochs, coordinate increments in the adopted coordinate system can be determined and the derived values computed.

Solutions using an orthogonal coordinate system are most often applied as the spatial basis for determination of structure deformations. In this case, the survey seeks to determine the $\mathrm{x}, \mathrm{y}, \mathrm{z}$ coordinates and changes are determined by the displacement vector components $\mathrm{dx}, \mathrm{dy}$ and $\mathrm{dz}$ computed from two successive survey periods. Indices referred to coordinate system planes or axes can also be computed based on the obtained results, e.g. linear distortion, shape distortion, angles of rotation, torsion and inclination.

In an orthogonal coordinate system (Fig. 2a), point position is determined as the common point of three planes and the values of the coordinates are the distances from the origin of the system to the respective planes. Changes in the position and basic geometric values describing the spatial arrangement of the elements of the examined structure can be determined based on individual elements of length, area, angles and volume. In an orthogonal coordinate system, the basic values measured with reference to coordinate system planes and axes are:

$$
\overrightarrow{d l}=d x \vec{i}_{x}+d y \vec{i}_{y}+d z \vec{i}_{z},
$$

called linear point position change,

$$
\begin{aligned}
& d S_{x}=d y d z, \\
& d S_{y}=d x d z, \\
& d S_{z}=d x d y,
\end{aligned}
$$

defining elementary surfaces in planes parallel to coordinate system planes and

$$
d V=d x d y d z
$$

defining elementary volume changes. 
(a)

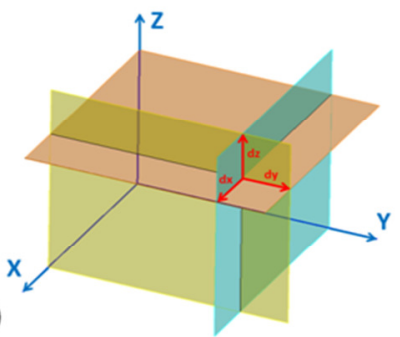

(b)

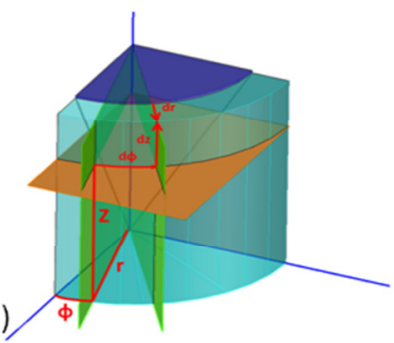

(c)

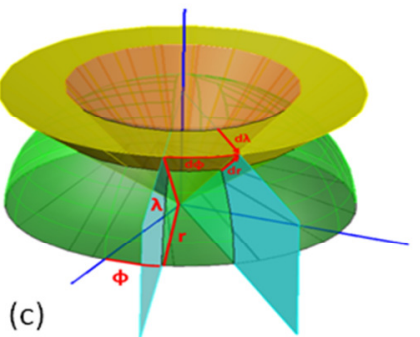

Fig. 2. Coordinate systems with point position change components: (a) orthogonal, (b) cylindrical, (c) spherical

In many cases, especially when an element of examination and analysis is a structure whose shape is a solid of revolution, it is convenient to determine the point position in a cylindrical coordinate system, in which the point position is defined by the r, $\varphi, z$ coordinates (Fig. 2b). Point coordinates are determined by the common point of the surface of a cylinder of radius $r$ and two planes: one passing through the $Z$ axis and forming the angle $\varphi$ with the XOZ plane and a plane parallel to the XOY plane distant from the origin of the system by the value Z. In this case, elementary changes in linear, surface elements and volume are expressed by the formulas:

$$
\overrightarrow{d l}=d r \vec{i}_{r}+r d \varphi \vec{i}_{\varphi}+d z \vec{i}_{z}
$$

called linear point position change,

$$
\begin{gathered}
d S_{r}=r d \varphi d z, \\
d S_{\varphi}=d r d z, \\
d S_{z}=r d r d \varphi,
\end{gathered}
$$

enabling the computation of elementary surface changes and

$$
d V=r d r d \varphi d z
$$

enabling the determination of elementary volume change.

Modern spatial data acquisition methods, e.g. laser scanners and mirrorless tacheometers, perform surveys in a spherical coordinate system, in which a point's position in space is determined by three coordinates r, $\varphi$, (Fig. 2c).

Point coordinates are defined by the common point of the surface of a sphere with radius $r$ and the centre at the origin of the system, the plane passing through the $\mathrm{Z}$ axis and forming the angle $\varphi$ with the $\mathrm{XOZ}$ plane and the surface of a cone with its vertex at the origin of the system and intersecting the XOY plane at the angle $\lambda$. In this situation, it is also convenient to handle survey results using a spherical system. The basic geometric elements which can be useful in structure evaluation are:

$$
\overrightarrow{d l}=d r \vec{i}_{r}+r d \lambda \vec{i}_{\lambda}+r \sin \lambda d \varphi \vec{i}_{\varphi}
$$

called linear point position change,

$$
\begin{gathered}
d S_{r}=r^{2} \sin \lambda d \lambda d \varphi, \\
d S_{\lambda}=r \sin \lambda d r d \varphi \\
d S_{\varphi}=r d r d \lambda
\end{gathered}
$$

enabling the computation of elementary surface changes and

$$
d V=r^{2} \sin \lambda d r d \lambda d \varphi
$$

enabling the determination of elementary volume change.

\section{Forms of visualization}

Presentation of the capabilities of CAD systems for visualization of construction displacements, requires the selection of suitable structures. Data completeness and the survey method determined the use of the results of control survey work for a residential building, a sports hall with a cylinder-shaped roof and a library with a dome-shaped roof. The preliminary stage of work relating to three-dimensional representation of displacements was the creation of three-dimensional models, by 
reproduction of their geometric configurations in a form consistent with the results of the first survey or consistent with the design.

Standard CAD system functions were used to find proper forms of representation and directions of observation, allowing clear documentation of changes. It was assumed after preliminary tests that it was advisable to separately distinguish two examined stages of structure changes because their joint representation causes considerable reduction in drawing clarity. The structure of the graphical database was designed to enable any compilation and representation of control survey results.

\section{Example 1. Dome-shaped roofing}

The roof covering of the Main Library of the University of Warmia and Mazury has a complex shape. One of its elements is a glass dome (Fig. 3). Because of the structure's inaccessibility and the material it was made of (glass, so a mirrorless survey cannot be applied), the survey was conducted using the method of tangent directions.

The survey results were used to determine the structure's position and shape by approximation of quadratic surfaces of revolution. The equation for a spherical surface assumes the form:

$$
F(x, y, z)=a_{11} x^{2}+a_{22} y^{2}+a_{33} z^{2}+2 a_{41} x+2 a_{42} y+2 a_{43} z+a_{44}=0 .
$$

The structure's coordinate system was transformed during result handling in such a way that the axis of rotation of the solid coincided with the $\mathrm{Z}$ axis of an orthogonal coordinate system.

(a)

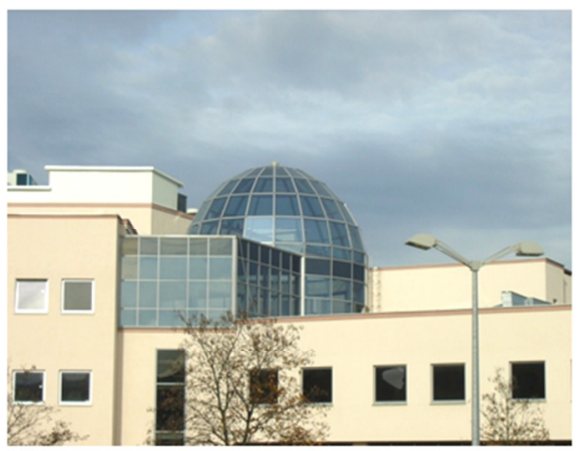

(b)

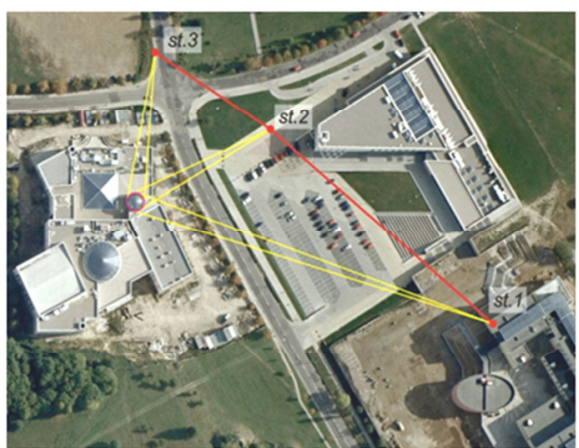

Fig. 3. Dome:(a) view from the south, (b) bird's-eye view of the structure with the control network shown

Determination of the $a_{i j}$ equation coefficients by the least squares method then enabled the computation of values describing the position and shape of the whole structure:

- coordinates of the centre of the main section $\left(\mathrm{x}_{0}, \mathrm{y}_{\mathrm{o}}, \mathrm{z}_{\mathrm{o}}\right)$,

- radius of the main section $(\mathrm{R})$,

- inclination of the axis of rotation of the approximated surface $(v)$,

- azimuth of the inclination of the axis of the approximated surface $\left(\mathrm{A}_{v}\right)$,

- and values characterizing the shape of the structure at the points where observations were performed:

- radial deviations $\left(\mathrm{v}_{\mathrm{r}}\right)$,

- tangential deviations $\left(\mathrm{v}_{\mathrm{s}}\right)$.

Defining radial and tangential deviations are most important from the point of view of evaluation of a dome-shaped structure. Their values are compiled in Table 1.

Table 1. Compilation of tangential and radial deviations

\begin{tabular}{llllll}
\hline Station & Level & \multicolumn{2}{c}{ Tangential deviations [m] } & \multicolumn{2}{c}{$\begin{array}{c}\text { Radial deviations [m] } \\
\text { Right }\end{array}$} \\
\hline 1 & Left & Right & 0,008 & 0,000 \\
& 1 & 0,126 & 0,126 & 0,019 & 0,007 \\
2 & 0,032 & 0,032 & 0,003 & $-0,014$ \\
& 3 & $-0,053$ & $-0,054$ & $-0,024$ & 0,020 \\
3 & 1 & 0,255 & 0,255 & $-0,092$ & $-0,014$ \\
& 2 & 0,125 & 0,124 & $-0,189$ & $-0,019$ \\
& 3 & 0,021 & 0,017 & 0,003 & 0,016 \\
& 1 & 0,212 & 0,212 & $-0,112$ & $-0,211$ \\
\hline
\end{tabular}


Two surfaces were generated as GRID models to visualize structure deformations - one based on the survey results (Fig. 4a) and the other approximated by a quadratic polynomial (Fig. 4b). The surfaces were created using standard functions of the ArcGIS application. Comparison of the surfaces and analysis of model differences enabled visualization of deviations of the examined structure from the spherical shape (Fig. 4c).

(a)

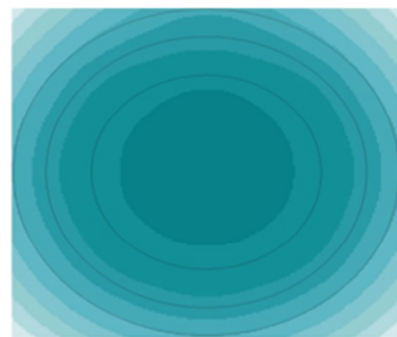

(b)

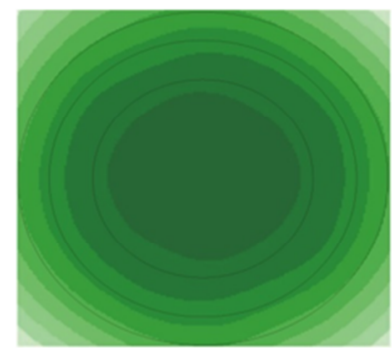

(c)

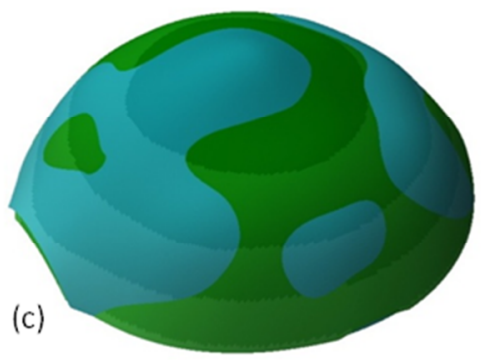

Fig. 4. Spatial visualization of the structure: (a) theoretical model, (b) approximated model, (c) superimposition of the models (a) and (b) in a 3D system

The values of radial and tangential deviations compiled in Table 1 enabled analysis of local deformations of the dome in the direction of the radius of horizontal sections and in directions tangent to these sections. TIN models showing the distribution of deformations were created based on the coordinates and the values of the deviations. Figure 5a shows the distribution of radial deviations with the statistics of the surface representing these deviations and Figure $5 \mathrm{~b}$ shows the corresponding illustration of tangential deviations.
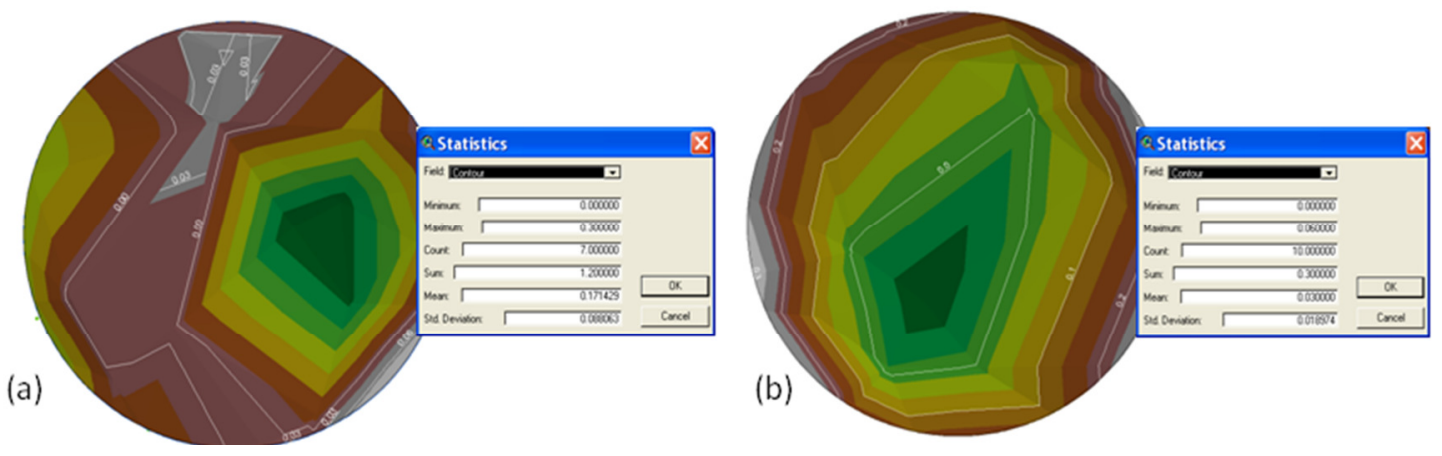

Fig. 5. Visualization of tangential deviations with model statistics: (a) radial, (b) tangential

\section{Example 2. Cylinder-shaped roofing}

The next structure in which the deviation from the theoretical shape was determined is the roof covering of a sports hall. This is a structure with a cylinder-shaped roof covering.
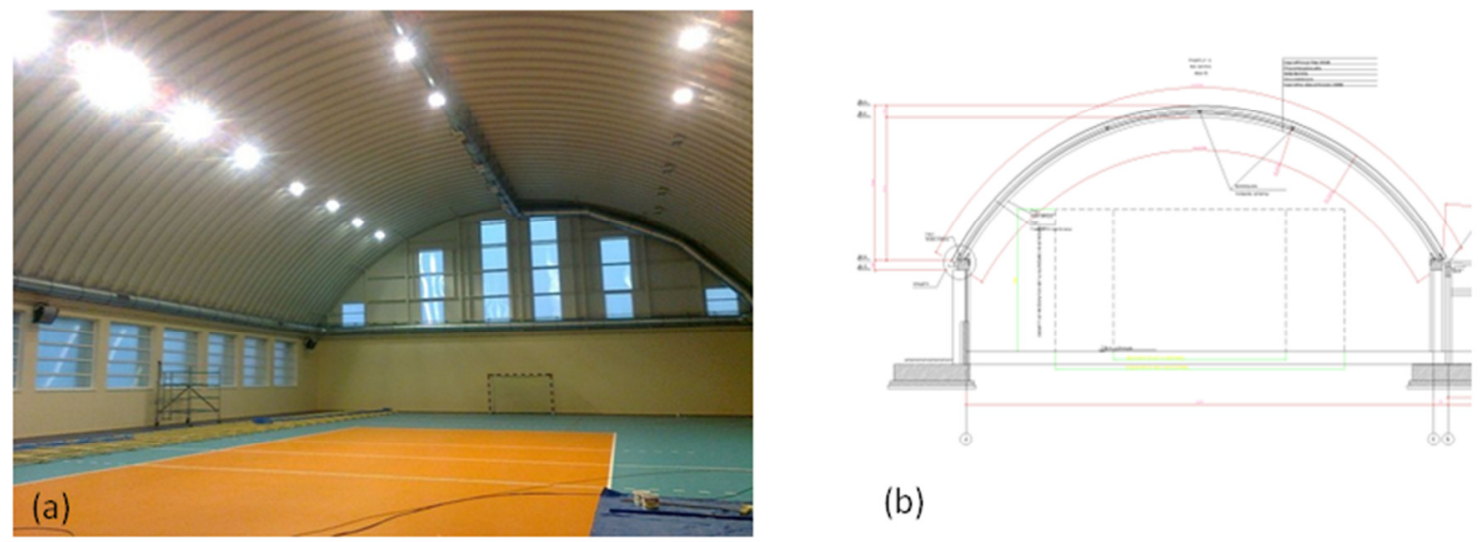

(b)

Fig. 6. Sports hall: (a) view from the inside, (b) cross-section

The survey control network consisted of two points. The line connecting the network points was in one vertical plane with the axis of rotation of the cylinder. The survey of the hall's roofing was conducted with a mirrorless tacheometer performing measurements in 5 sections. The cylinder's equation was determined by the least squares method based on the measured point coordinates. To facilitate computations, the coordinate system was adopted as illustrated in Figure 7. In this situation, the cylinder's sections in the X, Y plane and in parallel planes will be fragments of circles with the equation: 


$$
\left(X-x_{o}\right)^{2}+\left(Y-y_{o}\right)^{2}=R^{2},
$$

after transformation:

$$
X^{2}+Y^{2}-2 x_{o} X-2 y_{o} Y+x_{o}^{2}+y_{o}^{2}-R^{2}=0 .
$$

Adopting the denotations:

$$
\begin{gathered}
a=-2 x_{o}, \\
b=-2 y_{O}, \\
c=x_{o}^{2}+y_{o}^{2}-R^{2},
\end{gathered}
$$

we obtain:

$$
X^{2}+Y^{2}+a X+b Y+c=0 .
$$

Based on the survey set, observation equations can be formulated in the form:

$$
v_{i}=X_{j}^{2}+Y_{i}^{2}+a X_{i}+b Y_{i}+c,
$$

whose solution is the vector

$$
\left[\begin{array}{l}
a \\
b \\
c
\end{array}\right]=-\left(A^{T} A\right)^{-1} A^{T} \mathrm{~L} .
$$

The cylinder's parameters can be computed after computation of the equation coefficients, i.e.:

$$
\begin{gathered}
x_{o}=-\frac{a}{2}, \\
y_{o}=-\frac{a}{2}, \\
R=\sqrt{c-x_{o}^{2}+y_{o}^{2}} .
\end{gathered}
$$

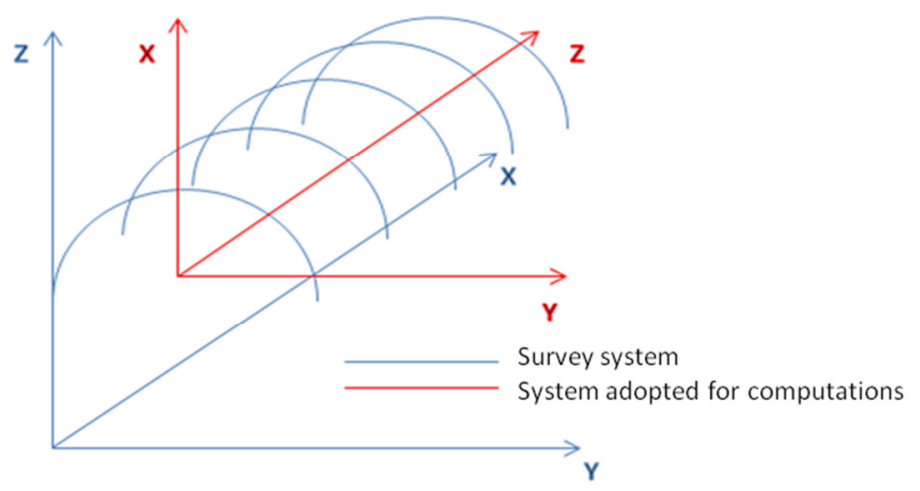

Fig. 7. Sports hall - adopted system for structure measurement and handling

The theoretical $\mathrm{Z}$ coordinates were generated at the points at which the survey was conducted based on the approximated cylinder's equation. Two sets of coordinates were thus obtained: the survey results and for the points of the surface of the theoretical cylinder. The X, Y coordinates of corresponding points are the same in both sets. Based on the point sets, point groups were created in the Autodesk Civil 3D system. They then became the basis for generation of TIN surfaces.

Using standard functions of the Civil 3D software, cross-sections were generated (Fig. 8) and an analysis was performed on the created models.

The cross-sections were created with a $1 \mathrm{~m}$ interval. Their visual evaluation allows the values of deviations to be assessed from the cylinder's shape. Precise measurements of deviations shown in the sections are also possible in the Civil 3D system. 

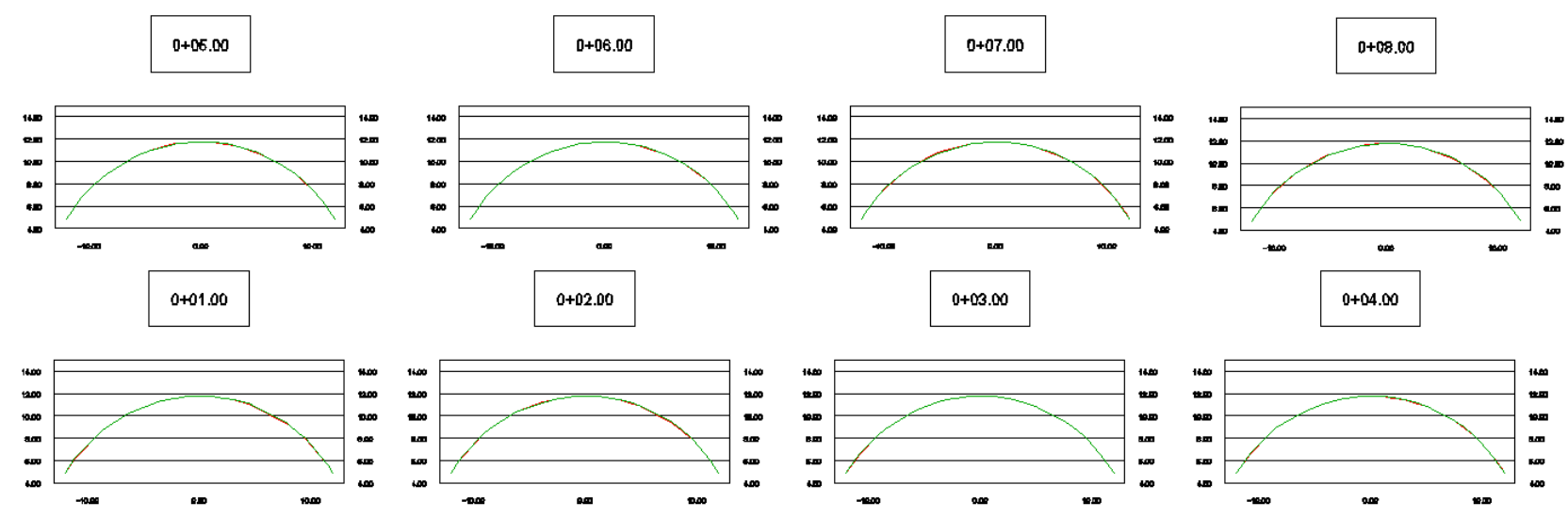

Fig. 8. Cross-sections of the sports hall's roofing

A more complete picture of deformations can be obtained by applying the so-called differential surface and using spatial analysis. A differential surface was created based on TIN models of the surface and was generated based on the approximated cylinder's equation, with the first of these models as the base surface and the second as the comparative surface. The generated surface adopted, as the $Z$ ordinates, deviations of the surface obtained based on the survey of the cylinder's shape adopted as that theoretically-determined along the $\mathrm{Z}$ axis.

The so-called ordinate analysis was performed to obtain an easily evaluable image of deviations. The analysis results, in the form of a hypsometric image with the legend, is shown in Figure 9a.

(a)

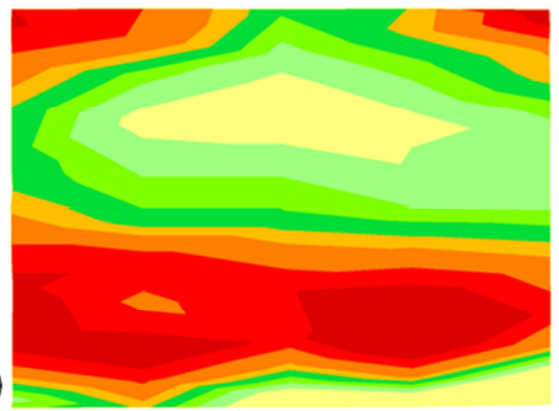

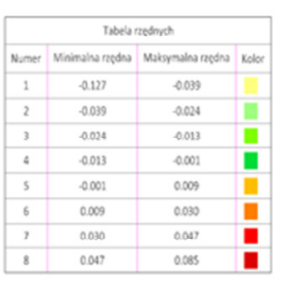

(b)

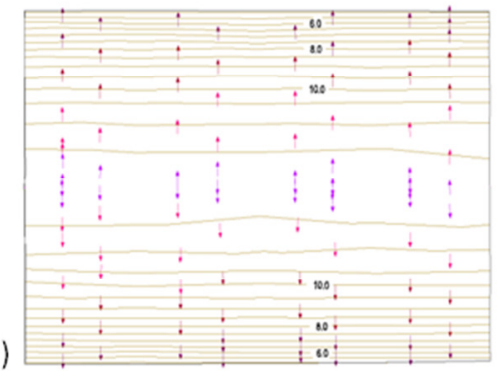

Fig. 9. Forms of visualization: (a) hypsometric model of vertical deviations, (b) analysis of slope vectors

From among the rich set of surface analyses offered by standard functions of the Civil 3D software, the analysis of slope vectors was selected, whose results, against the background of the contour configuration of the roofing, are shown in Figure $9 \mathrm{~b}$.

\section{Summary}

CAD and GIS systems are excellent tools for drawing up different forms of graphical representation and preparation of statistical compilations in the structure deformation examination process. They have particularly convenient tools for identification of the geometric relationships connecting individual elements of a structure, e.g. determination of the angle, distance, surface area, volume and the results of these actions can, in most cases, be automatically placed directly in the drawing. They are also an excellent tool for performing spatial analysis. This creates convenient conditions for compiling documentation, very often drawn up in control surveys, illustrating the values of deviations of examined elements from their designed or theoretical values.

\section{References}

[1] Bryś, H.; Przewłocki, S. 1998. Geodetic methods of structure displacement measurements. Warsaw: PWN Publishers.

[2] Lazzarini, T. et al. 1997. Geodetic measurements of displacements of structures and their surroundings. Warsaw: PPWK Publishers.

[3] Wójcik, M.; Wyczałek, I.; Sztukiewicz, W. 1989. Determination of spatial displacements of constructions and structures by the trigonometric method. Poznań: PPGK Geopoz Press.

[4] Bojarowski, K. 2011. Structure modelling in modernization of road and rail routes, UWM Scientific Bulletin 32: 6-14.

[5] Bojarowski, K.; Gościewski, D. 2011. Evaluation of structural components of structures using digital surface modelling, UWM Scientific Bulletin 32 16-27.

[6] Bojarowski, K.; Gościewski, D. Numerical maps in the designing and registration of engineering objects, Croatian Information Technology Society GIS Forum, 40-46.

[7] Bojarowski, K. 2006. Statistical and Spatial Analysis of Displacements, in Proceedings of the $12^{\text {th }}$ FIG Symposium on Deformation Measurements. Baden, Austria. 